\title{
SORORIDAD EN LOS PROCESOS DE RESISTENCIA A LAS PRÁCTICAS ARTÍSTICAS PRECARIAS
}

\section{Angélica García Estrada}

Escuela Nacional de Antropología e Historia (ENAH, México). Doctoranda

\section{Resumen}

La mayor parte de las prácticas artísticas en México son precarias, donde se gestan diversos procesos de resistencia. El presente artículo tiene como objetivos dar cuenta de las estrategias de resistencia que las artistas crean, de qué maneras inciden en los cuerpos y qué procesos afectivos se generan. Se realizó una etnografía a un grupo de mujeres artistas visuales de 32 a 42 años, donde se encontró que el trabajo colectivo es utilizado como una forma de resistencia, destacando en la dimensión emocional una sororidad que provee de un sentido de pertenencia, identidad, empatía y empoderamiento; y en los procesos de resistencia la imaginación, la creación y la transformación. Todos estos elementos son fuertemente influenciados por la postura feminista que tiene el grupo y han contribuido a que ellas generen otras resistencias, resistencias colectivas, resistencias sororas.

\section{Palabras clave: PRÁCTICAS ARTÍSTICAS; RESISTENCIAS; TRABAJO COLECTIVO; SORORIDAD; VÍNCULOS AFECTIVOS}

\section{SORORITY2 IN THE PROCESSES OF RESISTANCE TO PRECARIOUS ARTISTIC PRACTICES}

\begin{abstract}
Most of the artistic practices in Mexico are precarious, where various resistance processes are gestated. The purpose of this article is to give an account of the resistance strategies that artists create, how they affect the bodies and what affective processes are generated. An ethnography was performed on a group of women visual artists from 32 to 42 years old. It was found that collective work is used as a form of resistance, highlighting in the emotional dimension a sorority that provides a sense of belonging, identity, empathy and empowerment; and the processes of resistance are built by imagination, creation and transformation. All these elements are strongly influenced by the feminist position that the group has and have contributed to them generating other resistances, collective resistances, sororal resistances.
\end{abstract}

Keywords: ARTISTIC PRACTICES; RESISTANCES; COLLECTIVE WORK; SORORITY; AFFECTIVE BONDS

García Estrada, Angélica. 2019. "Sororidad en los procesos de resistencia a las prácticas artísticas precarias". AusArt 7 (2): 15-24. D0I: 10.1387 /ausart.21149

\section{AUSART}




\section{INTRODUCCIÓN}

La mayoría de las artistas visuales que trabajan en la Ciudad de México vive en condiciones de trabajo precarias que generan procesos de resistencia en ellas, que si bien no las saca de su situación precaria e inclusive puede conllevar a un mayor desgaste en el cuerpo, sí genera nuevas relaciones y procesos afectivos que le proveen de sentido y las empodera para crear otras resistencias colectivas. Este elemento emocional les dota de sentido, les da un lugar de pertenencia e identidad. El presente artículo ${ }^{3}$ tiene como objetivos dar cuenta de las estrategias de resistencia que las artistas generan, entre ellas la creación de espacios físicos donde se llevan a cabo prácticas sororas, de qué maneras inciden en los cuerpos y qué procesos afectivos se generan.

Por un lado, me parece imprescindible indagar en otras realidades de la escena artística como es la de las mujeres artistas ${ }^{4}$, y por otro recalcar, que se indaga de, desde (Citro 2009) y entre los cuerpos (Esteban 2004a) de las artistas ${ }^{5}$.

Este trabajo se centra en el cuerpo experiencial, que incluye las prácticas corporales y las representaciones, centrándome más en las primeras ${ }^{6}$.

\section{PRECARIEDAD Y FEMINISMO EN LAS PRÁCTICAS ARTÍSTICAS}

Una gran parte de las/los artistas visuales en la Ciudad de México vive una situación de precariedad laboral, no cuenta con un contrato social y lo que deriva de esto. El trabajo artístico se caracteriza por la inestabilidad, la flexibilidad, el pluriempleo, la multiactividad (Román 2016) y la autogestión ${ }^{7}$, lo que las/los lleva a buscar otras alternativas, generando estrategias de resistencia que en las mujeres artistas son más evidentes debido a las desigualdades de género ${ }^{8}$.

El grupo de artistas con quienes trabajo no está dentro del mainstream ${ }^{9}$ del arte, y por su condición de mujeres, tienen menos oportunidades para producir, distribuir y exponer su obra. Ya Linda Nochlin (1971) argumentaba en su famoso texto ¿Por qué no ha habido grandes mujeres artistas?, que las situaciones sociales e institucionales han impedido que el trabajo de las mujeres artistas florezca: "lo que las ha convertido en artistas con vocación de 
resistencia: resistir el cansancio producto de las horas de trabajo, las crisis económicas, las demandas familiares para poder producir" (Geat 2017, 287).

El feminismo tuvo un lugar crucial para la resistencia de las mujeres, utilizando el cuerpo y las emociones, permitiéndoles trabajar y producir desde otro lugar. ${ }^{10}$ A través de la experiencia incorporada (embodied) ${ }^{11}$ es que las artistas conectan con diversas emociones detonadoras de proyectos artísticos de resistencia.

El feminismo ${ }^{12}$ artístico hizo una importante revalorización de las artes tradicionales practicadas por las mujeres a lo largo de la historia ${ }^{13}$. Son prácticas que usualmente tienen que ver con una sororidad, que se hacen en grupo, las cuales fueron retomadas resignificándolas política y socialmente. El trabajar en colectividad y colaboración es uno de los pilares de las prácticas feministas, lo cual es imprescindible tener en cuenta ya que las artistas en el estudio son feministas y piensan, dicen, actúan y sienten ${ }^{14}$ como tal.

\section{METODOLOGÍA}

Se realizó una etnografía ${ }^{15}$ con seis mujeres artistas visuales que trabajan en la Ciudad de México en el 2018 y 2019. Se efectuaron tres entrevistas narrativas $^{16}$ a cada una de las colaboradoras, de una duración de 40 a 60 minutos cada una. Son cuatro mexicanas, una colombiana y una austriaca de edades entre los 32 y 42 años. Todas cuentan con una licenciatura en artes visuales o artes plásticas, cuatro tienen estudios de maestría y una cuenta con doctorado. Las seis se consideran feministas y trabajan con distintos medios, como gráfica, dibujo, textil, instalaciones, vídeo, performance, etc. Para todas ellas es muy importante el trabajo colectivo, aunque también tienen proyectos en lo individual.

Me enfoqué en el trabajo colectivo de las prácticas artísticas y lo que se genera en él: las emociones y resistencias y el consecutivo desgaste. Para fines analíticos destaco dentro de la dimensión emocional la construcción de una sororidad que les da un sentido de pertenencia, identidad, empatía y empoderamiento; y dentro de las resistencias destaco a la imaginación, la creación y la transformación. 
Cuatro de las participantes -Lisorix, Unorix, Nasorix y Nisorix- son parte de la misma colectiva, llamada Invasorix, pero a su vez están en otros colectivos. Las dos restantes -Helena y Deborah- han trabajado juntas y también forman parte de otros colectivos. Invasorix ${ }^{17}$ existe desde el 2013, tomando al alien como metáfora de alguien que no pertenece a este planeta, desafiando paradigmas sociales. No obstante que ellas no sienten pertenecer al circuito de arte hegemónico de la Ciudad de México, han creado un espacio de pertenencia para ellas y otrxs ${ }^{18}$. Para ellas esos otrxs son todas esas corporalidades que no son categorizadas dentro de lo normativo ${ }^{19}$. Como lo dice Lisorix, han tenido oportunidad de hacer pequeñas intromisiones que les gusta pensarlas como un ruido feminista que llega, hace disturbio y se retira, dejando desestabilizadas ciertas cosas que les interesa desestabilizar, como el patriarcado. Desean no solo desestabilizar al status quo sino al mundo del arte como lo conocemos. Denuncian las prácticas corruptas de las instancias culturales ${ }^{20}$ y tratan de crear espacios expositivos alternos ${ }^{21}$.

\section{EL DESGASTE ${ }^{22}$ DEL CUERPO}

Trabajar colectivamente, una de las propuestas feministas, es de suma importancia para las artistas, ya que lo vinculan con un compromiso político, y les confiere un sentido de pertenencia y de identidad. Pero para todas ha representado mucho más trabajo y desgaste del que tenían, pues por un lado no es fácil ponerse de acuerdo con tantas personas -no solo en cuanto a contenidos y formas sino también a tiempos y espacios- y por otro lado si bien les provee de inmensas satisfacciones, no les genera ingresos económicos ${ }^{23}$, o los pocos que pueden tener ${ }^{24}$ se lo tienen que dividir entre todas las integrantes del colectivo. Por ello tienen que buscar otros trabajos, la mayoría de ellos esporádicos, y/u otros proyectos, muchos de los cuales también son en colectividad. Como dice Lisorix: "tengo muchos colectivos y todos hacen poquitas cositas" 25 . Sin embargo, sin afán de romantizar este desgaste o inclusive a la resistencia, creo que en la práctica las artistas no ven a las colectividades como una bola de nieve, sino más bien como una constelación en expansión. En este sentido, las artistas formulan que hay que insistir en el trabajo colectivo. Unorix comenta: "Abrazar el trabajo colectivo es algo que nunca nos enseñaron. Hay que defenderlo y pelearlo. Nadie dijo que fuera fácil, pero se tiene que hacer. Y cuando haces algo con otras mujeres, hay algo que pasa que... te da amor". 
Se crea una sororidad con la que se cuidan y apoyan mutuamente, donde definitivamente para ellas lo más importante son los lazos afectivos que se tienen dentro, más que el trabajo que se tenga por delante. De aquí el amor del que habla Unorix, y así lo expresa Lisorix: "No tenemos tantos productos en el sentido de que los artistas tienen que estar produciendo todo el tiempo porque muchas veces nos atraviesa la vida, ¿no? Hay tantas contingencias en la vida que lo sano es atender a esas contingencias. Si una está deprimida, o tiene un pedo ${ }^{26}$ su familia, pues a lo mejor te ibas a ver para escribir una canción pero quizá es más urgente atender al ser humano, a la persona que tienes en frente, y eso también es un posicionamiento. Apostar por construir lazos, estrecharlos y no reproducir las violencias".

Mari Luz Esteban (2018) las llama comunidades o redes de apoyo mutuo ${ }^{27}$ y argumenta que los lazos afectivos que se generan en ellas han sido muy poco estudiados en antropología. Estamos hablando de lazos de amistad o amor tan importantes que los integrantes se consideran familia, de ahí que la autora, entre otros, proponga hacer una revisión de las teorías del parentesco ${ }^{28}$.

\section{RESISTENCIAS}

La resistencia nace en primer lugar en el cuerpo individual, es una acción que tiene como punto de partida la creación propia, pero después transita al cuerpo social y al cuerpo político ${ }^{29}$ donde adquiere más fuerza, donde puede generar verdaderos cambios y transformaciones. Hay una potencia y un deseo de imaginar, construir, desafiar y crear otras posibilidades de vida. Esto en sí mismo es una resistencia al status quo, además, se empoderan mutuamente creando más resistencias las cuales no se habrían dado en lo individual.

Los espacios físicos también son muy importantes para que estas resistencias y prácticas colaborativas se den. Un ejemplo es Pandeo, gestionado por Helena, quien lo describe de esta manera:

El principal objetivo de este proyecto es que a través del arte $y$ de las metodologías artísticas se promuevan otras formas de sociabilidad. Jugar con las etiquetas y las formas de ser sociales que están muy arraigadas, (...) poner a las personas que entran al espacio, incluso a los artistas, en la situación donde no pue- 
den comportarse como harían normalmente y tienen que hacerse conscientes y develar su corporalidad, ¿no?, y su performance en la vida (...) donde uno puede empezar a trabajar su agencia política. Entonces nosotros pensamos cómo podríamos tener la estética de la fiesta, y de ahí promover otras formas de pensar o crear ambientes donde personas que nunca se hubieran topado puedan tener una conversación, escucharse, llegar a conocerse o crear algo juntos.

Coincido con Muelas de Ayala $(2018,286)$ en que en estos espacios se da la "posibilidad para la creación de procesos colectivos que pueden favorecer el empoderamiento a través del placer y la diversión". Se deberían de generar y analizar más estos lugares, pues ahí se están gestando redes afectivas en un ambiente de placer y de goce, que son precisamente unos de los deseos feministas. Además, son ambientes de confianza, donde las/los miembros de la comunidad se cuidan mutuamente (no olvidemos la situación de violencia por la que cruza el país).

\section{CONCLUSIONES}

En este trabajo se muestra que en las colectividades gestadas en las prácticas artísticas se construye una sororidad que empodera a las integrantes, lo que genera más resistencias. Los procesos de resistencia se dan a través de la imaginación, la creación y la transformación. Las artistas imaginan otras posibilidades de existencia, lo que significa resistir a lo ya dado, a lo que nos presenta la sociedad; ellas crean otras formas de ser/estar en el mundo; y transforman a sus propios cuerpos y a las/los que trastocan con sus prácticas.

Los principales elementos con los que trabajan son lo emocional, lo sensible y lo lúdico, fundamentales en las prácticas artísticas. Son elementos que en sí mismos vinculan a las artistas cuando forman comunidad, que no siempre se dan en otros espacios. Estos elementos han sido un estandarte para las luchas feministas, abogando por la liberación y transformación de un cuerpo emocional/sensible, que tiene un conocimiento y que crea en/desde ahí. Con ellos se generan espacios de fiesta, goce y relajación, que permiten imaginar, crear y transformar desde un lugar de confianza, de seguridad, de amor. 
A pesar de que se piense que la tendencia actual apunta hacia el individualismo, este grupo de artistas demuestra que también se puede apostar por la colectividad, y lo vemos replicarse en muchos otros ámbitos laborales. Es algo que deberíamos buscar, practicar y enseñar.

Crear espacios de sororidad significa crear resistencias que construyen, no destruyen. Son espacios de respeto y aceptación donde no se reproducen las violencias. Son una posibilidad para la imaginación, creación y transformación de nuevos mundos. Considero muy importante tanto para el ámbito antropológico como para el artístico describir y analizar más estos espacios como generadores de conocimiento.

\section{Referencias bibliográficas}

Aschieri, Patricia. 2013. "Hacia una etnografía encarnada: la corporalidad del etnógrafo/a como dato en la investigación". X RAM- Reunión de Antropología del Mercosur- Situar, actuar e imaginar antropologías desde el Cono Sur. GT 11: 10 al 13 de julio de 2013, Córdoba, Argentina

Citro, Silvia. 2009. Cuerpos significantes: Travesías de una etnografía dialéctica. Buenos Aires: Biblos

Esteban Galarza, Mari Luz. 2004a. Antropología del cuerpo: Género, itinerarios corporales, identidad y cambio. Barcelona: Bellaterra

- . 2004b. "Antropología encarnada: Antropología desde una misma". Papeles del CEIC \#12

- . 2018. "Comunidades o redes de apoyo mutuo: Experiencias de mujeres feministas". En Etnografías feministas: Una mirada al siglo XXI desde la antropología vasca, Mari LuZ Esteban \& Jone M. Hernández García, coords., 361-82. Barcelona: Bellaterra

Geat, Andrea Soledad. 2017. "Resistencias femeninas: Arte contemporáneo regional desde una perspectiva de género". Revista de Investigaciones Feministas 8(1): 283-98

Gerber, Verónica \& Carla Pinochet. 2013. "Economías creativas y economías domésticas en el trabajo artístico joven". En Jóvenes creativos: Estrategias y redes culturales, Néstor García Canclini \& Ernesto Piedras Feria, coords., 129-54. Ciudad de México: Juan Pablos

González Abrisketa, Olatz \& Carlos García Grados. 2018. “¿Incorporar, encarnar, incorporar y/o corporear? Un ejercicio de traducción para pensar la agentividad de los cuerpos". En Etnografías feministas: Una mirada al siglo XXI desde la antropología vasca, Mari Luz Esteban \& Jone M. Hernández García, coords. 231-47. Barcelona: Bellaterra

Lipovetsky, Gilles \& Jean Serroy. 2013. La estetización del mundo: Vivir en la época del capitalismo artístico. Traducción de Antonio-Prometeo Moya. Barcelona: Anagrama

Lock, Margaret \& Nancy Scheper-Hughes. 1987. "The mindful body: A prolegomenon to future work in medical anthropology". Medical Anthropology Quarterly 1(1): 6-41

Muelas de Ayala, Laura. 2018. "Una mirada a las fiestas desde la antropología feminista: El placer como proceso creative y espacio político. En Etnografías feministas: Una mirada 
al siglo XXI desde la antropología vasca, Mari Luz Esteban \& Jone M. Hernández García, coords. 273-96. Barcelona: Bellatierra

Nochlin, Linda. (1971) 2008. “¿Por qué no ha habido grandes mujeres artistas?”. Traducción, Ana María García Kobeh. En Amazonas del arte nuevo, comisarios Josep Casamartina i Parassols \& Pablo Jiménez Burillo 283-89. Madrid: Fundación Mapfre

Ramírez Velázquez, Josefina. 2016. "Las emociones como categoría analítica en Antropología: Un reto epistemológico, metodológico y personal", en Cartografías emocionales: Las tramas de la teoría y la praxis, Oliva López \& Rocío Rosas, eds., 97-126. Ciudad de México: Universidad Autónoma de México

Ruido Pidre, María \& Jaron Rowan. 2007. "In the mood for work ¿Puede la representación alterar los procesos de valorización del trabajo cultural?". En Producta50: Una introducción a algunas de las relaciones que se dan entre la cultura y la economía, YProductions, ed.; Mari Paz Balibrea et al. Barcelona: Generalitat de Catalunya

\section{Notas}

${ }^{1}$ La sororidad es un concepto utilizado en algunos feminismos. Se refiere a una complicidad, una alianza, una hermandad entre mujeres.

${ }^{2}$ Sorority is a concept used in some feminisms. It refers to a complicity, an alliance, a sisterhood among women.

${ }^{3}\left({ }^{*}\right)$ Agradezco los comentarios y sugerencias al presente texto de Miren Urquijo, Gloria Estrada y Mónica Toledo.

Este trabajo es parte de mi investigación doctoral Cuerpos sensibles y en resistencia. Mujeres artistas visuales que trabajan en la Ciudad de México que efectúo en el posgrado de Antropología Física en la ENAH, México. Actualmente me encuentro realizando una estancia en la UPV/EHU bajo la tutoría de la Dra. Miren Urquijo.

${ }^{4}$ Como lo han hecho desde los setenta hasta ahora estudiosas feministas.

${ }^{5}$ Esto significa que no se estudia al cuerpo como un sujeto que no responde ni interactúa ante las circunstancias, sino se estudia en y desde el cuerpo, indagando en primera instancia en la propia corporalidad, y entre los cuerpos, en las relaciones que de ellos emanan. Así estamos hablando de un agente que actúa y responde de múltiples maneras ante el mundo.

${ }^{6}$ Se han vertido toneladas de páginas al estudio de las representaciones de las/los artistas, pero poco en sus prácticas. Es hasta recientemente (a partir de los años sesenta) que se ha tenido un interés por la agencia del artista y más específicamente a partir de los ochenta que se ha descrito y analizado su experiencia vivida.

${ }^{7}$ Las/los artistas no son los únicos en tener estas condiciones precarias, sin embargo, "(...) representan un modelo prototípico del trabajo en la era de la inseguridad social" (Gerber \& Pinochet 2013,152).

${ }^{8}$ Y como un ejemplo muy ilustrativo, una de las artistas, Nisorix, narró cómo en su primer día de clases, el maestro dijo que las mujeres eran artistas de 'clase $B$ '. Ella le exigió una explicación, a lo que él respondió que los hombres eran de 'clase $A$ ' y las mujeres ' $B$ ' porque ellas no se iban a dedicar al arte. El $60 \%$ del alumnado de la ENAP, la Escuela Nacional de Artes Plásticas de la UNAM, son mujeres, mientras que en los museos de la misma 
casa de estudios sólo el 30\% de las que exponen obra son mujeres.(Dirección General de Artes Visuales tendencias.cieg.unam.mx/boletín-14.html)

${ }^{(7}$ Las/los artistas visuales trabajan con diferentes medios, en un origen eran aquellas disciplinas donde lo visual era lo único a considerar, ahora todos los sentidos están involucrados (de hecho, los sentidos, en la práctica, no se separan). Dentro de las artes visuales están las bellas artes, las artes plásticas, las artes aplicadas, las artesanías, las artes digitales. Es un abanico extensísimo que comprende la pintura, dibujo, gráfica, escultura, instalación, grabado, textil, cerámica, ebanistería, vidrio, joyería, vídeo, sonido, performance, por mencionar algunas.)

${ }^{9}$ Moda o tendencia.

${ }^{10}$ El primer objetivo era descolonizar al cuerpo.

${ }^{11}$ Donde el cuerpo es el locus en donde la experiencia del mundo es vivida activamente. La palabra embodied ha sido traducida al castellano como incorporar, encarnar, incorporar, corporear, cada uno con diferentes implicaciones conceptuales (González \& García Grados 2018).

${ }^{12}$ Entiendo que no hay un feminismo sino múltiples feminismos, pero no es el objetivo de este artículo discutir ese tema, por lo que simplemente lo llamaré "feminismo".

${ }^{13}$ El bordado, el tejido, la cerámica, artesanías atribuidas a la mujer que generalmente se ejercían dentro de casa.

${ }^{14}$ El pensar-decir-hacer-sentir es un circuito experiencial que provee de sentido a los agentes (Ramírez 2016)

${ }^{15}$ Atendiendo a la dimensión corporal en el modo de estar-en-el-campo (Aschieri 2013, Esteban 2004).

16 Donde se pone la atención a la producción narrativa.

${ }^{17}$ Grupo de trabajo feminista cuir que hace canciones, vídeoclips, publicaciones DIY (Do It Yourself), lecturas de tarot y presentaciones performáticas. Un ejemplo de performance fue el corrido (canción mexicana que ensalza héroes) llamado "la justicia tiene caras" que cantaron el 13 de noviembre del 2018 frente a la Suprema Corte de Justicia, en el marco de "Emergencia. Puntos de dolor y resistencia en la Ciudad de México", proyecto que surgió a raíz de los feminicidios en México. Las artistas además convocaron a más artistas para que tocaran y cantaran juntas en memoria de algunas mujeres asesinadas en México. Este performance, como muchos otros, no tienen paga alguna, situación normalizada en la escena artística.

${ }^{18}$ Como quieren desafiar paradigmas de género también, no se quieren enunciar directamente como mujeres, les interesa anunciarse con la $X$, borrar un poco el género.

19 Es decir, dentro de lo que se dicta que "debe ser". Lo que está dentro de la norma, entendida como la regla o conjunto de reglas que condicionan las conductas de los individuos.

${ }^{20}$ Como las galerías de arte e instituciones gubernamentales, que tienen una descarada preferencia para exponer a artistas hombres, además de que cuando se hace una exposición en particular en los museos, se le paga a todos menos al artista (al curador, al museógrafo, al carpintero, el que monta la obra, el que pone el foco...)

${ }^{21}$ Un ejemplo de ello es Pandeo, una casa que gestiona Helena. En ella se organizan eventos de todo tipo (expos, performances, talleres, FIESTAS) donde se invitan a artistas que no tienen la oportunidad de entrar a los espacios expositivos hegemónicos y abierto a todo tipo de público, incluida la comunidad LGTTTB. 
${ }^{22}$ Me refiero al desgaste en el cuerpo relacionado con el trabajo, un estado de agotamiento físico y emocional que incide de varias maneras, como con fatiga, insomnio, tristeza, enojo o irratibilidad, falta de concentración, etc.

${ }^{23}$ Ojo, ellas no forman colectividades con la finalidad de tener ingresos económicos.

${ }^{24}$ La mayoría de las actividades que se generan en los colectivos son performances, canciones, acciones que involucran al activismo, lo cual no les representa ingresos.

${ }^{25} \mathrm{Y}$ una se podría preguntar, ¿por qué en vez de tener varios colectivos y hacer pocas cosas en cada uno, no tener uno solo y hacer más cosas? Las razones son varias, distingo dos: porque cada colectivo tiene diferentes objetivos y afinidades y la más importante por las redes que se generan en ellos.

${ }^{26}$ Un problema.

${ }^{27}$ Para Esteban las comunidades o redes de apoyo mutuo son "agrupaciones de personas (...) caracterizadas por hacer conjunto y el compartir elementos muy distintos: protección mutua, apoyo económico, material, psicológico y moral, actividades de mantenimiento de la vida cotidiana, cuidados relativos a la salud o a la crianza, o actividades de entretenimiento, sociales y políticas (...) basados en la reciprocidad y la solidaridad que funcionan de modo permanente" (Esteban, 2018:376).

${ }^{28}$ Ver Comunidades o redes de apoyo mutuo: Experiencias de mujeres feministas (Esteban 2018) donde la autora hace una revista de los sistemas de parentesco.

${ }^{29}$ Tomo como punto de partida al cuerpo individual, social y político de Margaret Lock y Nancy Scheper-Hughes (1987). El cuerpo individual es entendido en el sentido fenomenológico de la experiencia vivida de la experiencia corporal. El cuerpo social se refiere a los usos de la representación del cuerpo como un símbolo natural con el cual se piensa a la naturaleza, la sociedad y la cultura. El cuerpo político da cuenta de la regulación, vigilancia y control de los cuerpos. La estabilidad del cuerpo político descansa en su habilidad de regular las poblaciones (cuerpo social) y disciplinar los cuerpos individuales..

(Artículo recibido: 12-10-19; aceptado: 19-11-19) 